\title{
SOCS-3 is downregulated in progressive CKD patients and regulates proliferation in human renal proximal tubule cells in a STAT1/3 independent manner
}

\author{
Hannes Neuwirt ${ }^{1}$, Iris E Eder ${ }^{2}$, Martin Puhr ${ }^{2}$ and Michael Rudnicki ${ }^{1}$
}

Proliferation and the sequence of epithelial to mesenchymal transition (EMT) and mesenchymal to epithelial transition (MET), called epithelial-mesenchymal-epithelial (EME) cycling are pivotal mechanisms of kidney repair and fibrosis. Furthermore, data suggest that dedifferentiation (EMT) is a prerequisite for proliferation of tubule cells. These processes have been shown to be regulated by STAT1/3 signaling. Suppressor of cytokine signaling-3 (SOCS-3) is a negative regulator of STAT1/3 signaling. Using a transcriptomics data set of patients with proteinuric kidney diseases we found that low levels of SOCS-3 RNA were associated with high-serum creatinine values in the long-term follow-up, which suggested a role of SOCS-3, regulated signaling in progression of chronic kidney disease. This result was validated in an independent cohort of patients with proteinuric nephropathies on protein level. In addition $\sim 60 \%$ of STAT target genes were differentially expressed in relation to stable kidney disease patients. Using two renal cellular models and SOCS-3 knockdown by short interfering RNA we investigated SOCS-3 effects on oncostatin M-induced STAT activation, differentiation and proliferation. SOCS-3 knockdown resulted in enhanced PSTAT1/3 phosphorylation and epithelial differentiation. The latter effect was only slightly enhanced by OSM treatment. Cellular proliferation was inhibited after SOCS-3 knockdown. This effect could not be further stimulated by OSM. Effects of SOCS-3 knockdown were not enhanced by downregulation of STAT1/3, suggesting a STAT independent effect on cell cycle regulators. Indeed, knockdown and overexpression of SOCS-3 were associated with decrease and increase of cyclin D1, -E and proliferation, respectively. In summary, SOCS-3 inhibits phosphorylation of PSTAT1/3 in renal tubule cells. Additionally, we show for the first time that - in vivo-loss of SOCS-3 is associated with unfavorable prognosis. In vitro, downregulation of SOCS-3 inhibits dedifferentiation (EMT) and cellular proliferation in kidney proximal tubule cells.

Laboratory Investigation (2013) 93, 123-134; doi:10.1038/labinvest.2012.154; published online 29 October 2012

KEYWORDS: chronic kidney disease; cytokine signaling; progression; SOCS-3; STAT

The clinical course of chronic kidney disease (CKD) depends on the extent of renal tissue regeneration. It is widely accepted that renal prognosis depends much more on the degree of tubulointerstitial damage than on the kind or extent of glomerular injury. ${ }^{1}$ In this context renal tubule cells are not mere bystanders but seem to have an active role in the process of damage and regeneration. Thus, an expanding number of studies are published utilizing kidney cells of tubular origin in order to elucidate their role in these processes. It has been suggested that in a first step, tubular epithelial cells de-differentiate into myofibroblasts, ${ }^{2,3}$ which is accompanied by upregulation of mesenchymal markers such as alpha smooth muscle actin ( $\alpha \mathrm{SMA})$ or vimentin and loss of epithelial markers such as E-cadherin. ${ }^{4-8}$ This tubular dedifferentiation, which is called epithelial to mesenchymal transition (EMT), results in a reduced number of metabolic active tubule cells. This leads to an increased number of interstitial cells and is commonly considered as a marker of tubular cell injury. However, if correctly regulated, it also promotes cell survival and proliferation ${ }^{9-11}$ and provides the basis for renal tubular regeneration. ${ }^{10,12}$ Indeed increasing evidence suggests that dedifferentiation is a prerequisite for

\footnotetext{
${ }^{1}$ Department of Internal Medicine IV, Nephrology and Hypertension, Medical University Innsbruck, Innsbruck, Austria and ${ }^{2}$ Department of Urology, Medical University Innsbruck, Innsbruck, Austria

Correspondence: Dr H Neuwirt, MD, PhD, Department of Internal Medicine IV, Nephrology and Hypertension, Medical University Innsbruck, Anichstrasse 35, A-6020 Innsbruck, Austria

E-mail: Hannes.Neuwirt@i-med.ac.at

Received 6 March 2012; revised 4 September 2012; accepted 18 September 2012
} 
proliferation. ${ }^{13-16}$ Cellular growth can be induced by various mediators including cytokines or growth factors and is regulated by cyclin-dependent-kinases and cyclins, predominantly cyclin D1 and -E. After expansion of this less differentiated cellular pool, the second step of redifferentiation into epithelial cells occurs. This is called mesenchymal to epithelial transition (MET) and the sequence of EMT-MET is also known as epithelialmesenchymal-epithelial (EME) cycling.

Various cytokines, hormones and growth factors such as TGFß1 have been shown to influence proliferation and de-/ redifferentiation of tubular epithelial cells. ${ }^{17-20,21}$ Cytokineinduced signaling cascades have become a focus of interest of recent research and are controversially discussed. In the human kidney tubule cell line HK-2, OSM-induced cellular events are indicative for EMT. ${ }^{22}$ These effects were also associated with OSM-induced human proximal tubule cell scattering in three-dimensional collagen matrices. However, OSM might also exert antifibrotic effects. ${ }^{22}$ Thus, we hypothesize that profibrotic and antifibrotic effects of OSM are depending on either cell models or on the cellular context (eg regulation and/or expression status of regulators of cytokine siginaling). OSM was also shown to activate JAK/ STAT signaling. ${ }^{23,24}$ This was associated with reduced E-cadherin expression and induction of mesenchymal markers (eg fibronectin), suggesting an induction of EMT. In contrast, other studies reported that activation of STAT1 is crucial for epithelial differentiation. ${ }^{25}$ Moreover, activated STAT3 was shown to be implicated in 3D tubule formation (=epithelial differentiation) in renal proximal tubule cells (RPTCs). ${ }^{26,27}$ Additionally, various studies showed that STAT3 also induced proliferation in renal cells ${ }^{28-30}$ by upregulation of cyclin D1 and -E. ${ }^{28}$

Regulators of STAT signaling are the phosphatase SHP-1, protein inhibitors of activated STAT (PIAS) and suppressors of cytokine signaling (SOCS) proteins. ${ }^{31-35}$ The SOCS family consists of eight molecules (cytokine inducible SH2containing protein CIS and SOCS-1-7), which contain a variable amino-terminal region, a central $\mathrm{SH} 2$ domain, a conserved carboxy-terminal SOCS box and in case of SOCS-1 and -3 a kinase inhibitory region. These proteins are induced in response to several cytokines and growth factors. After binding to JAK and/or STAT, activation of STAT factors is inhibited. ${ }^{36}$ Viral overexpression of SOCS-3 and treatment with the JAK2/STAT inhibitor AG490 were published to mitigate renal interstitial fibrosis and damage. ${ }^{37,38}$ Knockdown of SOCS-3 increased STAT1 activation resulting in a more severe renal damage in a mouse model of Ang II infusion. ${ }^{39}$ We have previously shown that SOCS-3 inhibits activation of STAT1/3 and also has direct inhibitory effects on proliferation of prostate cancer cells. ${ }^{40-42}$

In this study, we investigated whether SOCS-3 may also be an important regulator of STAT1/3 signaling in renal tubule cells in vitro and in vivo. So far, only one study has investigated the expression of SOCS-3 in one kidney cell line $e^{43}$ but no study has been performed in human kidney biopsy material or using common cell lines, like HK-2 or LLC-PK1. In this study, we found that SOCS-3 is downregulated in patients with progressive decline of renal function. Moreover, we demonstrate that SOCS-3 knockdown enhances PSTAT1/3 signaling, stimulates epithelial differentiation and may have a role as a STAT1/3 independent regulator of proliferation of renal proximal tubule cells.

\section{MATERIALS AND METHODS \\ Patients Characteristics and Array Data}

We extracted SOCS-1/-3, SHP-1, PIAS 1-4 and STAT target gene expression values from a transcriptomics data set of laser-capture microdissected RPTCs isolated from biopsies of 16 patients with proteinuric nephropathies ${ }^{44,45}$ (Table 1). Array raw data, microarray protocols and detailed patient characteristics are accessible through the authors' website at www.microarray.at.

Owing to lack of material of the original cohort, immunofluorescence of SOCS-3 was performed in an independent cohort of kidney biopsies from 19 patients with vasculitis and IgA nephropathy (IGAN). Patients were defined as progressive $(\mathrm{P})$ when they reached the renal end point during follow-up (ie doubling of serum creatinine or reaching of end-stage renal disease (ESRD)). All other patients were defined as stable (S). Unaffected kidney tissues from individuals suffering from renal cell carcinoma treated with unilateral nephrectomy are referred to as healthy controls. Patient characteristics are summarized in Table 1. The Institutional Review Board of the University of Innsbruck accredited the use of surplus material from routine kidney biopsies for research purposes.

\section{Cell Culture and Chemicals}

LLC-PK1, HK-2 cells were obtained from the American Type Culture Collection (Rockville, MD). Cell lines derive from proximal tubule cells from human (HK-2) and swine (LLCPK1). Cell culture reagents were obtained from Gibco (Gibco Life Technologies, Lofer, Austria), OSM and doxycycline from Sigma (Vienna, Austria). Lipofectamine 2000 was purchased from Invitrogen (Lofer, Austria). HK-2 cells were cultured in keratinocyte-serum-free medium containing 5\% fetal calf serum, $5 \mathrm{ng} / \mathrm{ml}$ recombinant epidermal growth factor and $0.05 \mathrm{mg} / \mathrm{ml}$ bovine pituitary extract. LLC-PK1 cells were cultured in Eagle's MEM with 5\% FCS.

\section{Western Blot}

The protocol has been previously published. ${ }^{40}$ In brief, cells were washed with PBS and lysed. $50 \mu \mathrm{g}$ of protein per lane were resolved using 4 to 12\% Bis-Tris gels (Invitrogen, Leek, The Netherlands) and transferred onto a nitrocellulose membrane (Invitrogen). Primary antibodies were incubated at $4{ }^{\circ} \mathrm{C}$ over night. After washing, membranes were incubated with fluorescence-labeled secondary antibodies (Molecular Probes, Eugene, OR) and subsequently scanned and 
Table 1 Characteristics of patients in which renal SOCS-3 expression was assessed using A or IF

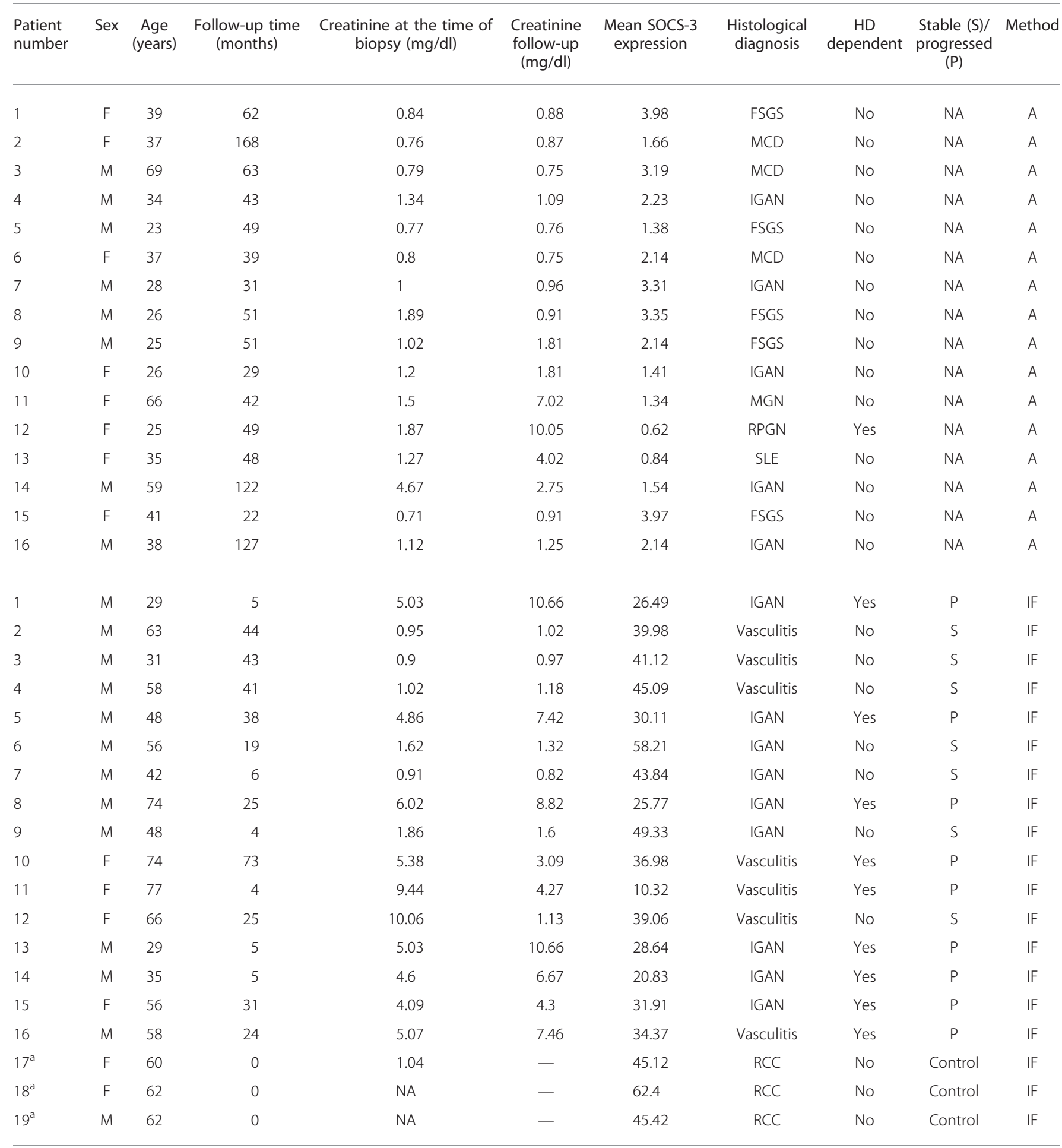

Abbreviations: A, microarray technology; FSGF, focal segmental glomerulosclerosis; IF, immunofluorescence; IGAN, IgA nephropathy; MCD, minimal change disease; RCC, renal cell carcinoma; RPGN, rapid progressive glomerulonephritis; SLE, systemic lupus erythematodes; vasculitis, ANCA-associated vasculitis. SOCS-3 expression from microarray data (A) is stated as ratio to reference RNA (Stratagene Universal Human Reference RNA, Agilent Technologies, Loveland, CO).

SOCS-3 protein expression as detected by IF was quantified using TissueFAXS (TissueGnostics, Vienna, Austria).

${ }^{a}$ Refers to patients with RCC in which non-tumor affected kidney tissue not adjacent to the tumor was used as control tissue. 
quantified using the Odyssey infrared imaging system (LiCor Biosciences, Lincoln, NE).

\section{qRT-PCR}

The detailed protocol and primer probe sequences have been previously published. ${ }^{40}$ In brief, after mRNA isolation, cDNA synthesis was performed using Superscript III RNase $\mathrm{H}$-reverse transcriptase (Invitrogen). PCR cycles were as follows: one cycle of denaturing at $95^{\circ} \mathrm{C}$ for $10 \mathrm{~min}$ followed by 40 cycles of $95^{\circ} \mathrm{C}$ for $15 \mathrm{~s}$ and $60^{\circ} \mathrm{C}$ for $1 \mathrm{~min}$.

\section{Short Interfering RNA Transfection}

The short interfering RNA (siRNA) sequence and detailed protocol used for targeting human SOCS-3 (si3) was previously published. ${ }^{46,47}$ On-target plus smart pool siRNAs targeting STAT1 and 3 were ordered from Dharmacon (Chicago, IL, Cat\# L-003544-00, L-003543-00). A nontargeting siRNA pool (siCo) (Dharmacon) served as negative control. In brief, two subsequent transfections were performed with Lipofectamine 2000 (Invitrogen) according to the manufacturer's protocol using $100 \mathrm{nMol} / \mathrm{l}$ siRNA against SOCS-3, or control siRNA during a period of 5 days. ${ }^{46}$

In case of experimental series with triple knockdown of SOCS-3, STAT1 and 3 the concentration of each siRNA was reduced to $33.3 \mathrm{nMol} / \mathrm{l}$ in order to yield $100 \mathrm{nMol} / \mathrm{l}$ as maximum siRNA concentration. In experiments using only STAT1/3 and SOCS-3 knockdown 33.3 and $66.6 \mathrm{nMol} / 1$ nontargeting siRNA pool was added in order to achieve the same concentration of $100 \mathrm{nMol} / \mathrm{l}$.

\section{Ectopic Overexpression of SOCS-3}

For inducible expression of N-terminal myc-tagged SOCS-3 in HK-2 cells, a tetracycline-responsive expression vector pBIG $2 \mathrm{i}^{48}$ containing the coding region of SOCS-3 and the myc tag was used. ${ }^{49}$ The plasmid was a kind gift of Prof. Wolfgang Doppler (Division of Medical Biochemistry, Innsbruck Medical University). A detailed protocol has been published previously. ${ }^{41}$ HK-2 cells were transfected with Lipofectamine 2000 (Invitrogen) according to the manufacturer's protocol. After $12 \mathrm{~h}$ doxycycline was added at a final concentration of $0.2 \mu \mathrm{g} / \mathrm{ml}$.

\section{${ }^{3} \mathrm{H}$-Thymidine Incorporation Assay}

The assay was carried out as reported previously. ${ }^{40,46}$ Subsequently $1 \mu \mathrm{Ci}$ of ${ }^{3} \mathrm{H}$-thymidine was added after stimulation of cells and radioactivity was quantified using Chameleon 5025 liquid scintillation counter (HVD Life Sciences, Vienna, Austria).

\section{Immunofluorescence}

Frozen sections of kidney biopsies or cell layers grown on 8well chambered cover glasses (Thermo Fisher Scientific, Cat\# 155409) were fixed in ice-cold acetone for $10 \mathrm{~min}$. After antigen retrieval and blocking, primary antibodies were added
(1:100 diluted in PBS). Secondary antibody was followed by embedding in VectaShield (Vector Laboratories, Burlingame, CA, USA) according to the manufacturer's protocol. Analysis was carried out using TissueFAXS (TissueGnostics, Vienna, Austria).

\section{Statistics}

Owing to non-Gaussian distribution non-parametric tests (Mann-Whitney-U, Kruskal-Wallis) were applied. P-values below 0.05 were defined as statistically significant and are encoded as follows: $P<0.05^{\star}, P<0.01^{\star *}, P<0.001^{\star * *}$. Nonstimulated cells served as control group except for experiments with SOCS-3 knockdown (si3). In these experiments asterisks indicate significant differences of SOCS-3 k.d. cells compared with the respective control with or without OSM treatment as indicated by horizontal bars, eg si3 $+10 \mathrm{ng} / \mathrm{ml}$ OSM is compared with siCo $+10 \mathrm{ng} / \mathrm{ml}$ OSM. Asterisks without bars indicate significant differences comparing OSM-treated and control cells.

\section{RESULTS}

\section{SOCS-3 is Negatively Correlated with Kidney} Function at Follow-Up In Vivo

In order to investigate a potential role of STAT signaling itself and its negative regulators in vivo we extracted SOCS-1/-3, SHP-1, PIAS 1-4, STAT target gene and in addition upstream regulators of STAT signaling (JAK1-3, TYK2) expression data out of a published microarray data set of laser-capture microdissected proximal tubule cells isolated from $16 \mathrm{CKD}$ patients with proteinuric renal diseases (Table 1). ${ }^{44,45}$ Renal SOCS-3 expression was significantly diminished in patients with low kidney function (and respective high creatinine levels) at follow-up (Pearson- $R=-0.614, \quad P=0.011$, Figure 1a) (median follow-up: 49 months, range 22-168). Because of the wide range of follow-up periods we also correlated SOCS-3 expression to the delta-Creatinine/year (calculated by: ((Creatinine at biopsy - Creatinine at follow-up)/ (follow-up period in months/12))), thereby confirming a significant negative correlation between SOCS-3 and deltacreatinine/year (Pearson- $R=-0.588, P=0.025$ ). Interestingly, SOCS-3 did not correlate with clinical parameters such as diagnosis, histological damage, creatinine at biopsy (Pearson- $R=-0.288, P=0.279$ ), proteinuria at biopsy or proteinuria at follow-up. Eleven of 21 (52.4\%) STAT target genes (Supplementary Table 1) were up- or downregulated by $>1.5$ or $<0.5$-fold, respectively. Approximately $64 \%$ of those $(7 / 11)$ were significantly regulated $(P<0.05)$, suggesting a role of STAT signaling in those patients. However, concerning the upstream molecules (JAKs, TYK2) and most negative STAT regulators (SHP-1, PIAS1-4, SOCS-1) we did not find any significant alterations.

Next, we stratified the patient cohort according to SOCS-3 expression levels in groups above or below the median SOCS-3 expression $($ median $=1.025)$. Patients with SOCS-3 
a
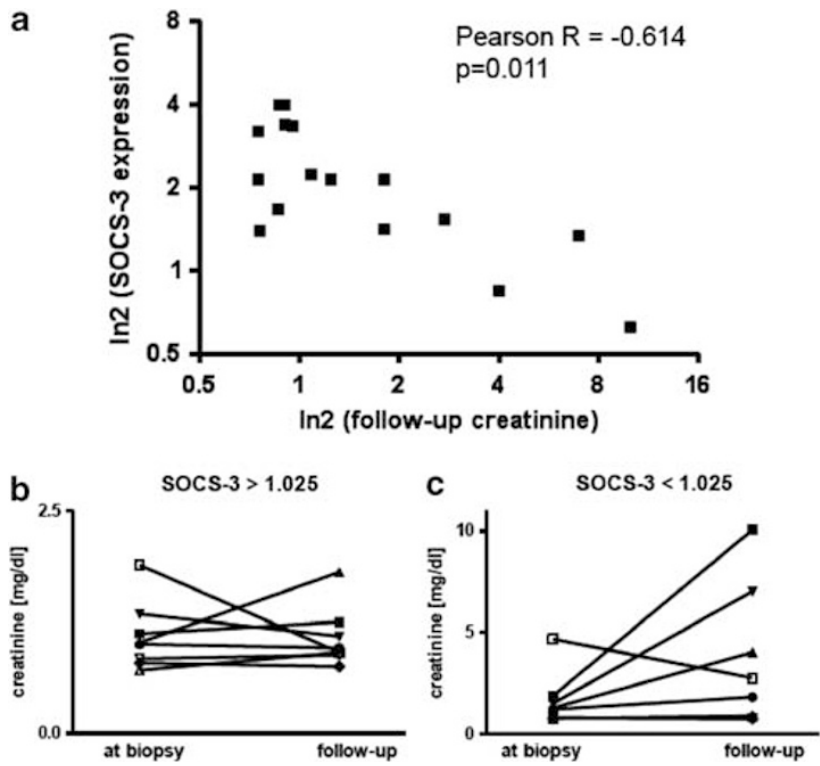

Figure 1 Suppressor of cytokine signaling (SOCS)-3 mRNA levels correlate with kidney function at follow-up in vivo. SOCS-3 mRNA expression levels were extracted from microarray data set derived from microdissected renal proximal tubule cellss of 16 chronic kidney disease (CKD) patients. (a) SOCS-3 expression in CKD patients negatively correlates with follow-up creatinine. Median values are shown as 3 SOCS-3 spots were found on the array chips. (b, c) Patients are grouped according to their SOCS-3 expression levels above or below median $(=1.025)$. In patients with low SOCS-3 levels serum creatinine remains stable during follow-up.

expression above median did not develop a progressive kidney disease (Figure $1 \mathrm{~b} / \mathrm{c}$ ).

\section{SOCS-3 is Significantly Downregulated in Patients with ESRD}

Our results from the mRNA expression experiments suggested a role of SOCS-3 and STAT1/3 signaling in kidney proximal tubule cells during progression of chronic kidney disease. This is of importance because the renal prognosis depends much more on the degree of tubulointerstitial damage than on the kind or extent of glomerular injury, even in glomerular diseases. ${ }^{1}$ Next we evaluated also the protein expression of SOCS-3 in renal biopsies. For this purpose we used cryo-cut sections from routine kidney biopsies from an independent cohort of 19 patients suffering from IGAN $(n=9)$, anti-nuclear cytoplasmatic antibody (ANCA) associated vasculitis $(n=7)$ and healthy individuals $(n=3)$ (Table 1). The median follow-up was 25 months (range: $5-73)$. SOCS-3 expression was found predominantly in the cytoplasm of tubule cells with highest levels in patients with preserved kidney function at time of biopsy (Figure 2). Patients who were on dialysis at time of diagnosis and who did not recover during follow-up had substantially diminished renal SOCS-3 protein expression (controls/S vs $\mathrm{P}-\mathrm{P}=0.00029)$. The Pearson correlation coefficient of SOCS-3 expression levels and creatinine at time of biopsy
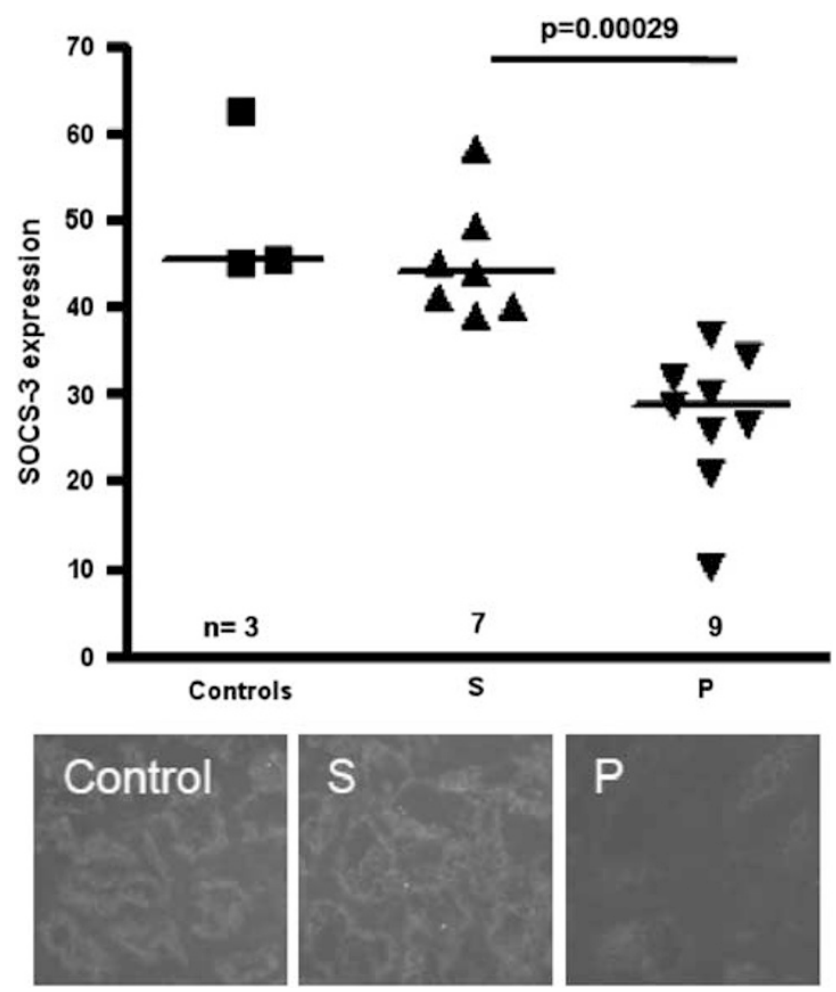

Figure 2 Suppressor of cytokine signaling (SOCS)-3 immunofluorescence in human kidney samples. Nineteen patient samples (three controls, seven chronic kidney disease (CKD) patients with stable disease (S), nine patients with progressed CKD (P)) were analyzed for SOCS-3 expression levels. Neg Co refers to isotype control (Dako). Upper panel depicts quantitative analysis of SOCS-3 expression as assessed by TissueFAXS (TissueGnostics, Vienna, Austria). Lower panel shows representative photographs of each group.

and follow-up was $-0.516 \quad(P=0.041)$ and -0.617 $(P=0.011)$, respectively. Of note, patient nr. 12 , who had dialysis dependent renal failure at time of biopsy but recovered during follow-up, exhibited high SOCS-3 protein levels. These results suggest that low SOCS-3 mRNA and protein levels in renal tubules are associated with an unfavorable renal prognosis.

\section{SOCS-3 Knockdown Enhances STAT1 and STAT3 Phosphorylation}

As SOCS-3 has been shown to negatively regulate STAT1 and -3 phosphorylation we next asked whether downregulation of SOCS-3 has any influence on STAT1/3 signaling and subsequent cellular functions like proliferation and differentiation in kidney proximal tubule cells. For these in vitro studies, we utilized two proximal tubular cell lines, HK-2 and LLC-PK1 and assessed the expression of SOCS-3 on mRNA and protein level by qRT-PCR, western blot and immunofluorescence. SOCS-3 was expressed in both investigated cell lines at mRNA and at protein level. Basal SOCS-3 mRNA and protein expression was higher in LLC-PK1 compared with HK-2 cells. Immunofluorescence confirmed higher 

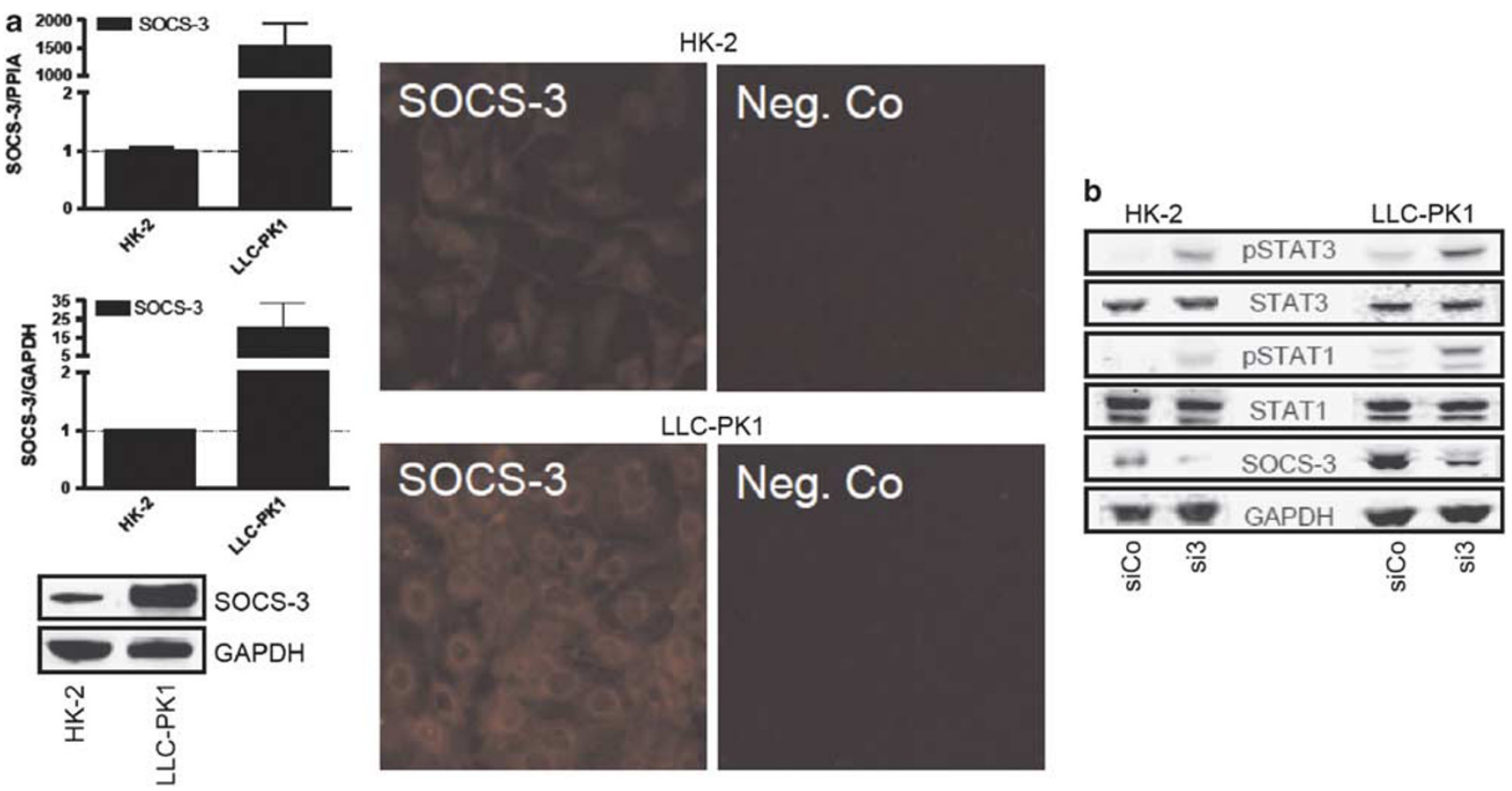

Figure 3 Suppressor of cytokine signaling (SOCS)-3 is expressed in kidney proximal tubule cells and influences STAT1/3 phosphorylation. (a) LLCPK-1 express higher SOCS-3 protein and mRNA compared with HK-2 cells. Cells were grown either in chambered cover glasses or in 6-well plates and basal expression levels of SOCS-3 mRNA and protein were assessed after $72 \mathrm{~h}$ using qRT-PCR and western blot/immunofluorescence, respectively.

Means + s.d. of nine independent experiments are shown. (b) SOCS-3 knockdown causes increased basal activity of pSTAT1 and 3. SOCS-3 was knocked down using short interfering RNA (100 nmol/I) (si3) in LLC-PK1 and HK-2 cells. Under these conditions STAT1/3 phosphorylation, levels of SOCS-3 and GAPDH were assessed and compared with cells transfected with a non-targeting pool (Dharmacon) (siCo). Representative western blots are shown; experiments were performed three times in LLC-PK1 and four times in HK-2 cells.

expression in LLC-PK1 cells (Figure 3a). Consistent with literature, SOCS-3 staining was cytoplasmatic and perinuclear. ${ }^{40}$ Next, we characterized STAT1/3 phosphorylation under standard culture conditions after knockdown of SOCS-3 (SOCS-3 k.d.) using specific siRNA (si3) compared with a non-targeting siRNA pool (siCo). SOCS-3 k.d. induced basal phosphorylation of STAT1 and STAT3 in both cell lines (Figure 3b).

As OSM stimulates STAT1 and STAT3 signaling $^{22,24}$ and was also shown to affect de- and redifferentiation (EMT and MET) in various cellular models including kidney tubule cells, we used this cytokine to study regulation of ligandstimulated phosphorylation of STAT1/3. In both cell lines we detected an OSM-induced concentration- and timedependent phosphorylation of STAT1 and STAT3 (Figure 4a). Furthermore, upregulation of pSTAT1/3 by OSM was significantly enhanced in both cell lines after SOCS-3 k.d. (Figure 4b), suggesting that SOCS-3 is implicated in inhibition of basal and of OSM-stimulated STAT1/3 phosphorylation.

\section{SOCS-3 Downregulation Effects RPTC Differentiation}

In order to study whether or not an altered SOCS-3 expression affects epithelial differentiation, we studied effects of SOCS-3 k.d. on E-cadherin, vimentin and $\alpha$ SMA cell marker expression. LLC-PK1 and HK-2 cells were transfected as explained above but OSM treatment was prolonged to $72 \mathrm{~h}$. In LLC-PK1 cells (Figure 5, upper panel) OSM treatment alone increased expression of the epithelial marker E-cadherin, whereas mesenchymal markers vimentin and $\alpha$ SMA were slightly downregulated. Most importantly, knockdown of SOCS-3 alone (without OSM) significantly upregulated E-cadherin, and significantly downregulated the expression of the mesenchymal markers vimentin and $\alpha \mathrm{SMA}$. These effects were only moderately enhanced by OSM.

In HK-2 cells (Figure 5 lower panel) SOCS-3 k.d. alone also yielded a significant upregulation of E-cadherin and downregulation of both mesenchymal markers. Similarly to LLC-PK1 cells, these changes were only moderately enhanced with simultaneous OSM treatment. OSM single treatment slightly decreased E-cadherin and increased vimentin expression, which is consistent with literature. ${ }^{22,50,51}$ aSMA was not changed after OSM treatment alone. These results indicate that knockdown of SOCS-3 may induce epithelial differentiation in proximal tubular cells. These changes were associated with increased phosphorylation of STAT1 and 3 in si3, OSM and si3 + OSM-treated cells (Figure 4a and b).

\section{SOCS-3 Knockdown Inhibits Proliferation of RPTCs}

Renal repair and recovery, eg after acute kidney injury depends - at least in part-on proliferation of tubular epithelial cells. ${ }^{52}$ Thus, we next elucidated the effects of SOCS-3 

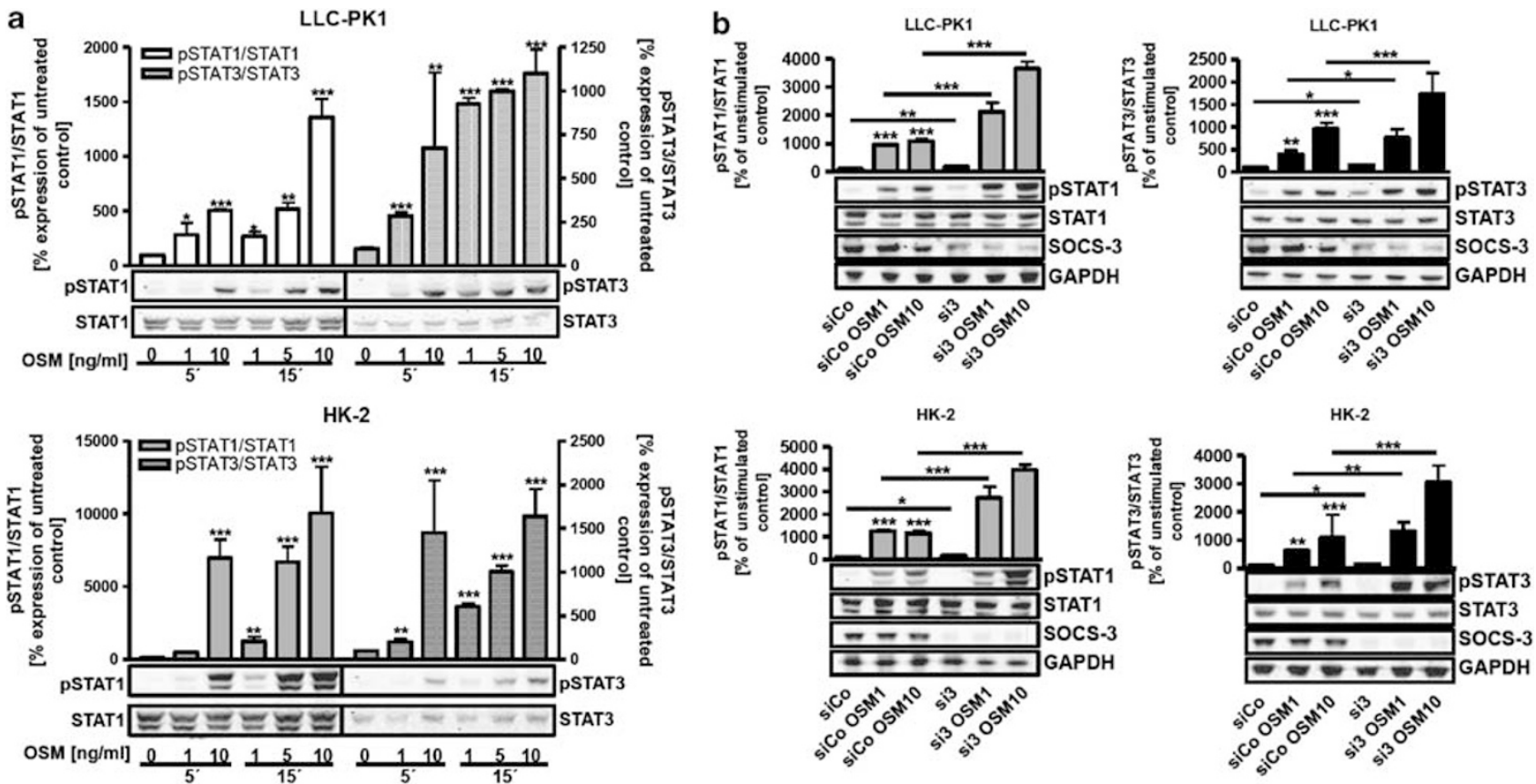

Figure 4 Suppressor of cytokine signaling (SOCS)-3 influences OSM-induced activation of STAT1 and 3. (a) LLC-PK1 and HK-2 cells were plated in sixwell plates and treated with OSM for the assigned time periods with two different concentrations (1,5 and $10 \mathrm{ng} / \mathrm{ml})$. A time- and dose-dependent increase of phosphorylated isoforms of STAT1 and 3 was found in both cell lines, as assessed by western blotting. Means + s.d. of six independent experiments are shown. (b) LLC-PK1 and HK-2 cells were transfected either with short interfering RNA (siRNA) against SOCS-3 (si3) or a non-targeting siRNA (Dharmacon) (siCo) and stimulated with OSM ( 1 and $10 \mathrm{ng} / \mathrm{ml}$ ) for $15 \mathrm{~min}$. Both STAT1 and 3 activation upon OSM stimulation were substantially increased after si3 treatment. Means + s.d. of at least five experiments are shown. Of note, asterisk mark significant changes in si3 w/o OSM compared with siCo w/o OSM-treated cells (eg siCo vs si3; siCo $+1 \mathrm{ng} / \mathrm{ml} \mathrm{OSM}$ vs si3 $+1 \mathrm{ng} / \mathrm{ml}$ OSM).

knockdown on proximal tubular cell proliferation (Figure 6). In LLC-PK1 and in HK-2 cells OSM treatment caused a significant increase of ${ }^{3} \mathrm{H}$-thymidine incorporation. Interestingly, SOCS-3 k.d. itself already inhibited proliferation, which was not significantly altered by OSM treatment compared with the respective control (eg siCo $+10 \mathrm{ng} / \mathrm{ml}$ OSM $v s$ $\mathrm{si3}+10 \mathrm{ng} / \mathrm{ml} \mathrm{OSM}$ ) (Figure 6, left panel).

To rule out a possible induction of apoptosis by SOCS-3 k.d. we measured caspase $3 / 7,-8$ and -9 activity after OSM treatment with and without SOCS-3 k.d. Our results show that neither treatment was associated with induction of apoptosis (Supplementary Figure 1). Therefore, we next measured levels of cell cycle regulatory molecules (cyclin E, D1, cdk2, -4). Cdk2 and -4 were not significantly altered in any treatment (data not shown). OSM stimulation alone increased cyclin E and -D1 levels although cyclin E only slightly and not significantly in LLC-PK1 cells (Figure 6, right panel). Similar to our previous experiments, SOCS-3 k.d. alone already resulted in a downregulation of cyclin $\mathrm{E}$ in LLC-PK1 cells and cyclin D1 in HK-2 cells, which could not be further stimulated by OSM treatment. In LLC-PK1 cells cyclin D1 was slightly induced by OSM besides si3 treatment, although this stimulation was only very moderate. In HK-2 cells similar results were found for cyclin E. These results suggest an inhibition of proliferation by knockdown of SOCS-3, which might be a direct effect of SOCS-3 independent of STAT1/3 phosphorylation.

Hence, we next measured cellular growth after STAT1/3 knockdown in combination with SOCS-3 k.d. (triple knockdown, Figure 7). In line with our previous experiments (Figure 6), knockdown of STAT1 and 3 (siST1/3) resulted in an inhibition of proliferation (data not shown). SOCS-3 k.d. (si3) alone, as shown above, again reduced cellular growth. Finally, triple knockdown of STAT1/3 and SOCS-3 (siST1/ $3+$ si3) did not yield any further growth inhibitory effect compared with single treatments. Most importantly, OSM treatment did not further change proliferation in any treatment group except controls, where ${ }^{3} \mathrm{H}$-thymidine was induced (see also Figure 6), although phosphorylation was increased in all treatment groups (Figure 7). These results suggest that SOCS-3 might inhibit cellular proliferation at least via a STAT1/3 independent mechanism.

\section{SOCS-3 Overexpression Induced Proliferation and Upregulation of Cyclin D1 and -E}

Hence, we next asked whether SOCS-3 overexpression might induce proliferation in HK-2 cells. As shown in Figure 8, SOCS-3 overexpression indeed enhanced cellular growth. 


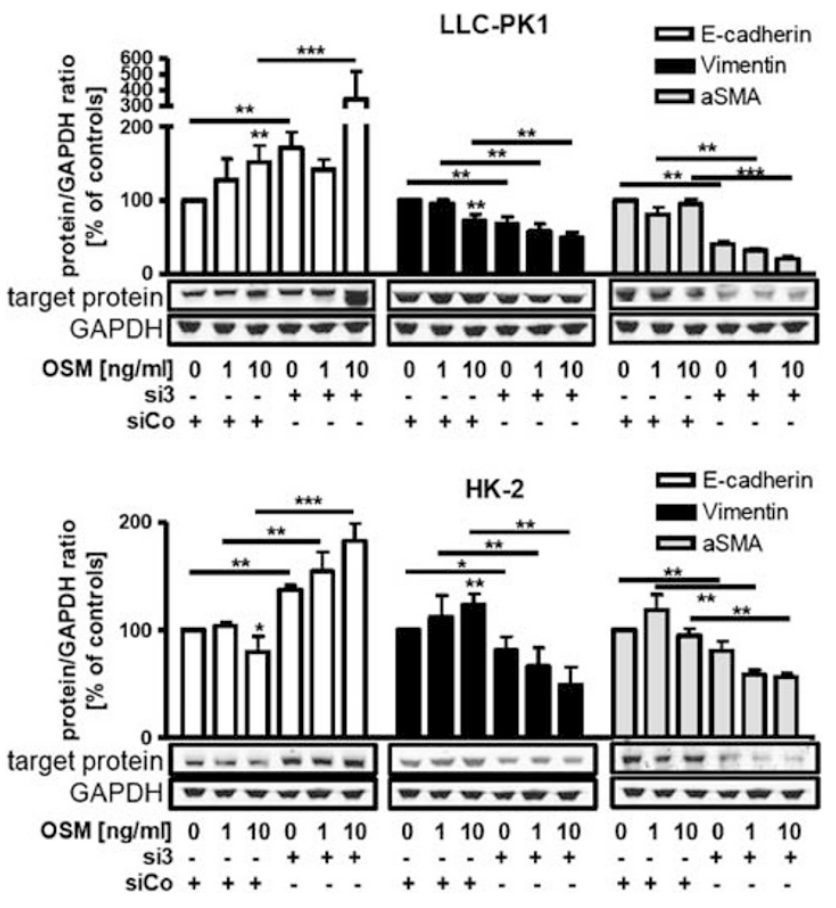

Figure 5 Impact of suppressor of cytokine signaling (SOCS)-3 modulation on the differentiation markers E-cadherin, vimentin and alpha smooth muscle actin. LLC-PK1 and HK-2 cells were transfected as described in section 'Materials and Methods' and subjected to $72 \mathrm{~h}$ of OSM treatment ( 1 and $10 \mathrm{ng} / \mathrm{ml}$ ). Graphs show quantitative values of proteins and representative western blots, respectively. SOCS-3 knockdown enhances OSM-stimulated epithelial differentiation. Means + s.d. of at least four independent experiments are shown.
In order to further investigate this finding, we looked at the expression of ckd2, -4, as well as cyclin D1 and -E. We found that SOCS-3-induced proliferation was associated with an upregulation of cyclin D1 and -E, while cdk2 and -4 were not altered.

In conclusion, our data suggest that SOCS-3 itself acts as an inducer of proliferation. Furthermore, SOCS-3 has a role as negative regulator of OSM-induced STAT1 and 3 phosphorylation. OSM-induced STAT1/3 activation enhances proliferation by induction of cyclin D1 and -E. This OSMinduced stimulation of cell proliferation can be abrogated by downregulation of SOCS-3 in a STAT1/3 independent manner. On the other hand SOCS-3 overexpression stimulates cell division by upregulation of cyclin $\mathrm{D} 1$ and $-\mathrm{E}$ suggesting a direct growth stimulatory effect of SOCS-3 (Figures 6-8).

\section{DISCUSSION}

The sequence of dedifferentiation (EMT), which is usually considered as deleterious, and redifferentiation (MET), which may be beneficial, is called EME cycling. ${ }^{10,12}$ This process provides a basis for proliferation and regeneration of the kidney tubule system. STAT1 and 3 have been shown to be implicated in all these cellular processes. The aim of our study was to characterize SOCS-3 expression in vivo and its impact on STAT1/3 signaling in renal tubule cell function in vitro. The reason for investigating this molecules in kidney tubule cells was that the extend of tubular damage correlates
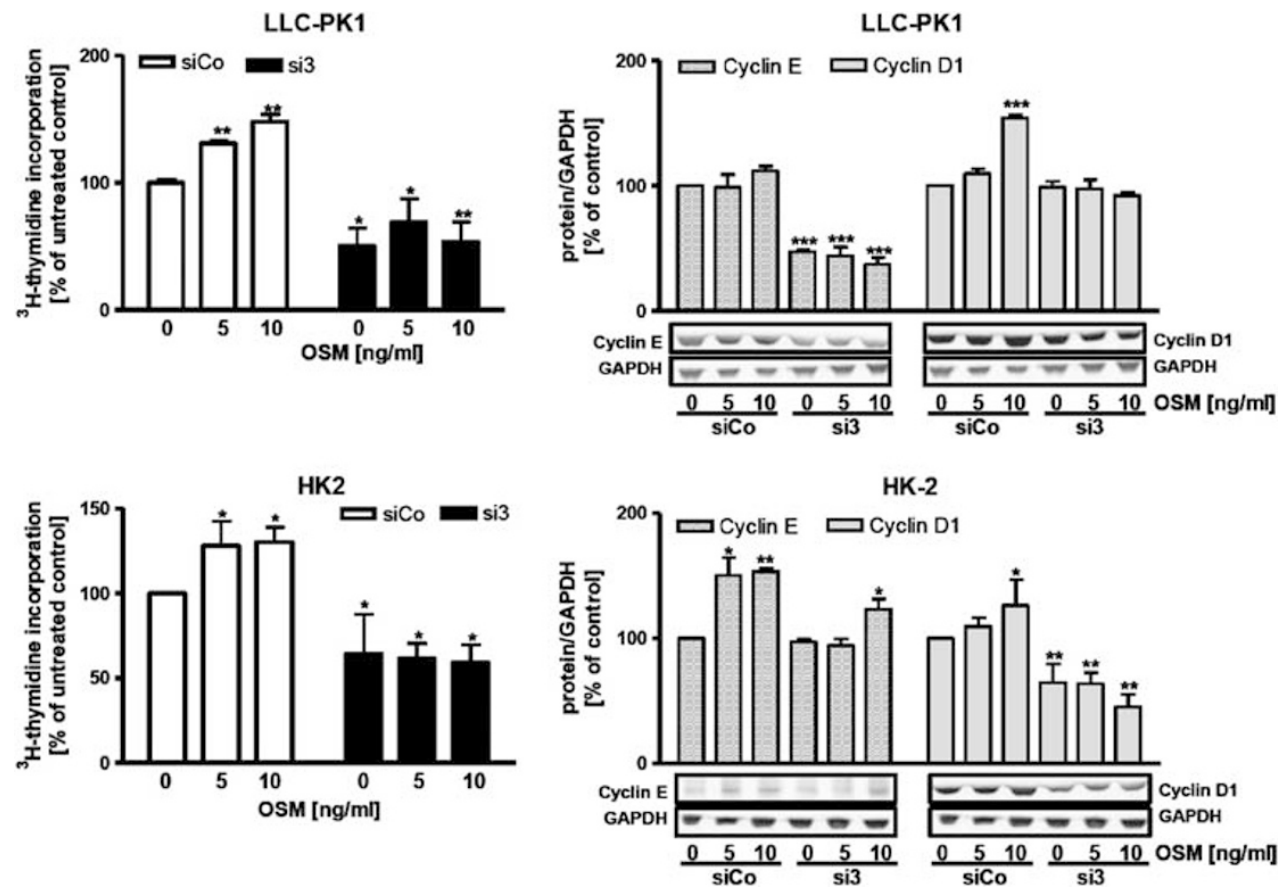

Figure 6 Effects of suppressor of cytokine signaling (SOCS)-3 on proliferation w/o OSM stimulation. Left panel: LLC-PK1 and HK-2 cells are growth stimulated by OSM (after $48 \mathrm{~h}$ ) as assessed by ${ }^{3} \mathrm{H}$-thymidine incorporation, which is abrogated by knockdown of SOCS-3. Of note, asterisks mark significant changes in si3 w/o OSM compared with siCo w/o OSM-treated cells (eg siCo vs si3; siCo $+1 \mathrm{ng} / \mathrm{ml}$ OSM vs si3 $+1 \mathrm{ng} / \mathrm{ml}$ OSM). Right panel: Upregulation of cyclins by OSM was abrogated by simultaneous si3 treatment. Means + s.d. of five independent experiments are shown. 

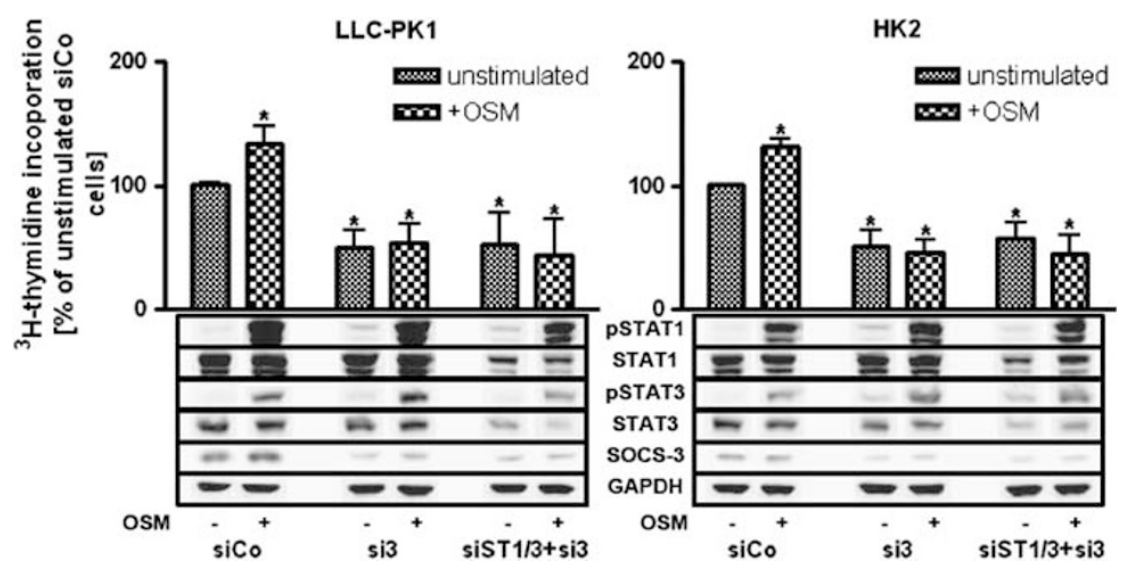

Figure 7 Triple knockdown of STAT1/3 and suppressor of cytokine signaling (SOCS)-3. LLC-PK1 and HK-2 cells are growth, transfected with a nontargeting short interfering RNA (siRNA) pool, specific STAT1 and 3 (siST1/3) (Dharmacon) or SOCS-3 siRNA (si3). ${ }^{3} \mathrm{H}$-thymidine incorporation was measured after $48 \mathrm{~h}$ of OSM or vehicle treatment. Additionally western blots were carried out in order to assess expression status of unphosphorylated STAT1/3, SOCS-3 and GAPDH. Means + s.d. of three independent experiments are shown.

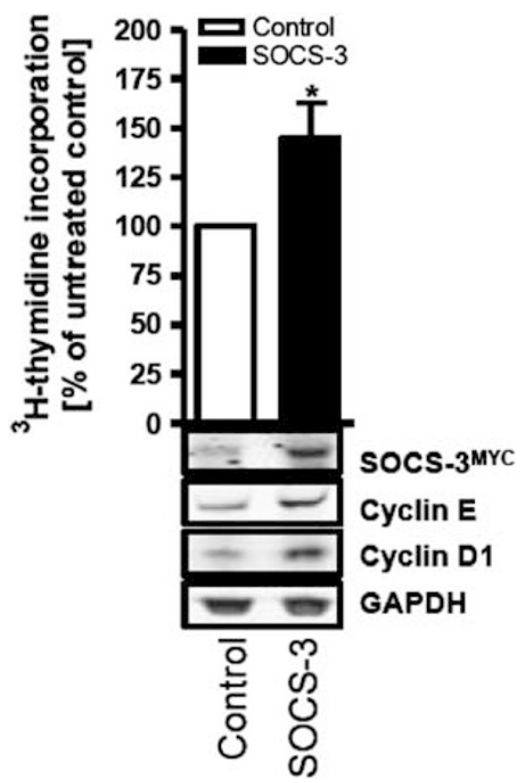

Figure 8 Ectopic overexpression of suppressor of cytokine signaling (SOCS)-3 enhanced proliferation and expression of cyclin D1 and -E as assessed by western blot. Means + s.d. of four independent experiments are shown.

better than glomerular changes with prognosis concerning kidney function, even in glomerular diseases. ${ }^{1}$

We started with a transcriptomics data set of microdissected proximal tubule cells of patients with proteinuric nephropathies and revealed that SOCS-3 levels at time of biopsy were downregulated in patients with high creatinine at follow-up (Pearson- $R=-0.614, P=0.011$, Figure 1a). Similarly, a significant negative correlation was found when SOCS-3 levels were correlated with change of renal function per year. In addition, 52\% STAT target genes were differentially regulated (64\% of which statistically significant), whereas upstream regulators or other negative regulators of STAT signaling, besides SOCS-3, were not significantly altered. This suggested a role of STAT $1 / 3$, which is negatively regulated by SOCS-3 in these patients. Consistent with this finding it was shown that PSTAT3 is upregulated in kidneys from patients suffering from lupus nephritis, IGAN and ANCA-associated vasculitis compared with normal kidney. ${ }^{30}$

Next, we investigated SOCS-3 protein expression in kidney biopsy specimens in a second cohort of patients and found SOCS-3 expressed in tubule cells with a cytoplasmatic expression pattern. Additionally, SOCS-3 expression was strongly reduced in patients with impaired kidney function/ high-serum creatinine at follow-up (Figure 2) (Pearson$R=-0.761, P=0.0026)$. This is consistent with our microarray data reported above (Figure 1). In particular, ESRD patients exhibited significantly lower SOCS-3 levels compared with those with only impaired kidney function and stable creatinine during the follow-up period $(P=0.0022)$. Of note, knockdown of SOCS-3 yielded more severe renal damage associated with an upregulation of PSTAT1 in a mouse model of angiotensin II infusion. ${ }^{39}$ Concerning the loss of SOCS-3 in ESRD patients, no studies in renal cells or kidney disease exist. However, there is evidence that SOCS-3 is lost in squamous cell carcinoma ${ }^{53}$ or lung cancer ${ }^{54}$ due to promoter hypermethylation.

In vitro we found SOCS-3 expressed on mRNA and protein levels in proximal tubule LLC-PK1 and HK-2 cells. OSMinduced STAT1/3 phosphorylation and knockdown of SOCS-3 by siRNA (si3) enhanced this effect (Figures 3 and 4). Furthermore, we investigated the impact of SOCS-3 k.d. alone and in combination with OSM treatment on differentiation markers. Consistent with literature, OSM stimulation alone downregulated E-cadherin and slightly induced vimentin in HK-2 cells, suggesting induction of EMT. ${ }^{22,50,51}$ 
However, knockdown of SOCS-3 alone caused changes suggestive for epithelial differentiation and these were slightly intensified after combination with OSM treatment (Figure 5). A study by Liu et al. ${ }^{43}$ has also investigated the role of SOCS-3 in a human proximal tubule cell line. In their experiments, OSM induced EMT and this was enhanced by SOCS-3 knockdown. However, cell culture media and siRNA sequences, and most importantly the cell line used differ from those in our study. Furthermore, those authors used a different panel of differentiation markers (eg CK18, collagen type I) in order to investigate EMT induction. To the best of our knowledge, no study has reported of an inductive effect of SOCS-3 knockdown on epithelial differentiation in kidney proximal tubule cells. Additionally, in our hands this effect was only slightly induced by co-stimulation with OSM and subsequent phosphorylation of STAT1/3. Thus, we believe that this may in part be a direct effect of SOCS-3.

In vivo, inhibition of SOCS-3 by antisense oligonucleotides in rats yielded more severe renal damage. ${ }^{39}$ Barasch et al. ${ }^{55}$ showed that leukemia inhibitory factor, an inducer of STAT3, was shown to induce epithelial differentiation in rat kidneys. Boccaccio et al. ${ }^{56}$ have shown that hepatocyte growth factorinduced tubulogenesis of epithelial cells was STAT3 dependent. Additionally, a study published recently suggested that OSM-induced pSTAT1 and/or 3 might contribute to tubulointerstitial protection as TGF $\beta 1$ induced matricellular proteins were inhibited by concomitant treatment with OSM. ${ }^{23}$ However, it is possible that induction or stabilization of an epithelial phenotype decreases regenerative capacity by deterioration of EME cycling (EMT-MET sequence).

Other studies suggested that inhibition of STAT signaling by administration of AG490 ${ }^{57}$ or adenoviral overexpression of SOCS $-3^{38}$ significantly reduced renal lesions such as glomerulosclerosis and interstitial fibrosis, respectively. Still, the methods used in these studies did not specifically inhibit STAT signaling in proximal tubule cells but in virtually all cells, including those of the immune system. This might limit the information concerning the role of SOCS-3/STAT signaling specifically in proximal tubule cells..$^{58,59}$

As renal repair and recovery also depend on the proliferative capacity of tubular epithelial cells ${ }^{52}$ we elucidated the impact of SOCS-3 modulated STAT signaling on proliferation. OSM treatment induced pSTAT1/3, expression of cyclin D1 and -E and cellular proliferation (Figure 6). Consistent with these findings phosphorylated STAT3 was shown to induce proliferation in RPTCs ${ }^{28}$ and renal cancer cells. ${ }^{29}$ Arakawa et al..$^{30}$ have shown that pSTAT3 is correlated with cell proliferation in human glomerulonephritis. Zhang et al. ${ }^{28}$ have published recently that pSTAT3-induced proliferation in renal proximal tubule cells. Consistent with our results this effect was mediated by upregulation of cyclin D1 and E.

However, SOCS-3 knockdown alone already inhibited cellular growth. In addition, si3-mediated growth inhibition could not be compensated by OSM. Simultaneous downregulation of SOCS-3, STAT1 and -3 did not further enhance the effects of SOCS-3 k.d. alone (Figure 7). Most importantly these results did not change after stimulation with OSM, although phosphorylation of STAT1 and 3 was induced, indicating that SOCS-3 may act as growth stimulatory mediator at least in a STAT1/3 independent manner. Thus, we next overexpressed SOCS-3 in order to assess a direct effect of SOCS-3 on proliferation and found that SOCS-3 overexpression induced cyclin D1 and -E protein levels and cellular growth (Figure 8).

In summary, we show that loss of SOCS-3, which acts as an inducer of proliferation in our in vitro experiments, abrogates the growth stimulatory effect of STAT1/3 signaling. This finding is similar to a study carried out in prostate cancer cells where knockdown of SOCS-3 together with db-cAMP treatment also yielded a decrease of cellular growth. ${ }^{40}$

In conclusion, in vivo, SOCS-3 is significantly downregulated in patients with decline of kidney function. Furthermore, we report that knockdown of SOCS-3 increased basal, as well as OSM-induced phosphorylation of STAT1 and 3. SOCS-3 knockdown induced epithelial differentiation. Combined treatment with OSM and SOCS-3 k.d. yielded only slightly stronger effects. Moreover, SOCS-3 itself may induce proliferation as indicated by SOCS-3 overexpression experiments, and-consistent with this-SOCS-3 knockdown yields in an abrogation of OSM-stimulated proliferation. These effects seem to be STAT $1 / 3$ independent. As recently reviewed 'tubular cells can repair kidney damage [... ] by dedifferentiation and proliferating. ${ }^{13,14}$ Importantly, dedifferentiation seems to be a prerequisite for kidney tubule cell proliferation. ${ }^{15,16}$ Thus, we hypothesize that low levels of SOCS-3 in CKD patients may cause inhibition of dedifferentiation (and EME cycling) and proliferation, both of which are fundamental mechanisms of kidney repair and regeneration, finally resulting in progressive disease. Supplementary Information accompanies the paper on the Laboratory
Investigation website (http://www.laboratoryinvestigation.org)

\section{ACKNOWLEDGEMENTS}

We thank Prof. Gert Mayer and Prof. Herbert Schramek for critical review of an early version of the manuscript and Prof. Wolfgang Doppler for providing the pBIG2i-Vector for SOCS-3 overexpression. This work was funded by the Tyrolean Wissenschaftsfonds. Array data available at www.microarray.at

\section{DISCLOSURE/CONFLICT OF INTEREST}

The authors declare no conflict of interest.

1. Nath KA. Tubulointerstitial changes as a major determinant in the progression of renal damage. Am J Kidney Dis 1992;20:1-17.

2. Strutz F, Okada H, Lo CW, et al. Identification and characterization of a fibroblast marker: FSP1. J Cell Biol 1995;130:393-405.

3. Iwano M, Plieth D, Danoff TM, et al. Evidence that fibroblasts derive from epithelium during tissue fibrosis. J Clin Invest 2002;110:341-350. 
4. Bedi S, Vidyasagar A, Djamali A. Epithelial-to-mesenchymal transition and chronic allograft tubulointerstitial fibrosis. Transplant Rev (Orlando) 2008:22:1-5.

5. Burns WC, Thomas MC. The molecular mediators of type 2 epithelial to mesenchymal transition (EMT) and their role in renal pathophysiology. Expert Rev Mol Med 2010;12:e17.

6. Zeisberg M, Kalluri R. The role of epithelial-to-mesenchymal transition in renal fibrosis. J Mol Med 2004;82:175-181.

7. Jinde K, Nikolic-Paterson DJ, Huang XR, et al. Tubular phenotypic change in progressive tubulointerstitial fibrosis in human glomerulonephritis. Am J Kidney Dis 2001;38:761-769.

8. Rastaldi MP, Ferrario F, Giardino $L$, et al. Epithelial-mesenchymal transition of tubular epithelial cells in human renal biopsies. Kidney Int 2002;62:137-146.

9. Mene $\mathrm{P}$, Polci $\mathrm{R}$, Festuccia F. Mechanisms of repair after kidney injury J Nephrol 2003;16:186-195.

10. Sharples EJ. Acute kidney injury: stimulation of repair. Curr Opin Crit Care 2007;13:652-655.

11. Cochrane AL, Kett MM, Samuel CS, et al. Renal structural and functional repair in a mouse model of reversal of uretera obstruction. J Am Soc Nephrol 2005;16:3623-3630.

12. Ishibe S, Cantley LG. Epithelial-mesenchymal-epithelial cycling in kidney repair. Curr Opin Nephrol Hypertens 2008;17:379-385.

13. Humphreys BD, Valerius MT, Kobayashi A, et al. Intrinsic epithelial cells repair the kidney after injury. Cell Stem Cell 2008;2:284-291.

14. Little MH. Renal organogenesis: what can it tell us about renal repair and regeneration? Organogenesis 2011;7:229-241.

15. Vogetseder A, Picard N, Gaspert A, et al. Proliferation capacity of the renal proximal tubule involves the bulk of differentiated epithelia cells. Am J Physiol Cell Physiol 2008;294:C22-C28.

16. Witzgall R, Brown D, Schwarz C, et al. Localization of proliferating cell nuclear antigen, vimentin, c-Fos, and clusterin in the postischemic kidney. Evidence for a heterogenous genetic response among nephron segments, and a large pool of mitotically active and dedifferentiated cells. J Clin Invest 1994;93:2175-2188.

17. Aresu L, Rastaldi MP, Pregel P, et al. Dog as model for downexpression of E-cadherin and beta-catenin in tubular epithelial cells in renal fibrosis. Virchows Arch 2008:453:617-625.

18. Aresu L, Rastaldi MP, Scanziani E, et al. Epithelial-mesenchymal transition (EMT) of renal tubular cells in canine glomerulonephritis. Virchows Arch 2007;451:937-942.

19. Aroeira LS, Aguilera A, Sanchez-Tomero JA, et al. Epithelial to mesenchymal transition and peritoneal membrane failure in peritoneal dialysis patients: pathologic significance and potential therapeutic interventions. J Am Soc Nephrol 2007;18:2004-2013.

20. Wynn TA. Cellular and molecular mechanisms of fibrosis. J Pathol 2008;214:199-210.

21. Liu Y. Epithelial to mesenchymal transition in renal fibrogenesis: pathologic significance, molecular mechanism, and therapeutic intervention. J Am Soc Nephrol 2004;15:1-12.

22. Pollack V, Sarkozi R, Banki Z, et al. Oncostatin M-induced effects on EMT in human proximal tubular cells: differential role of ERK signaling. Am J Physiol Renal Physiol 2007;293:F1714-F1726.

23. Sarkozi R, Hauser C, Noppert SJ, et al. Oncostatin M is a novel inhibitor of TGF-\{beta\}1-induced matricellular protein expression. Am J Physiol Renal Physiol 2011;301:F1014-F1025.

24. Nightingale J, Patel S, Suzuki N, et al. Oncostatin M, a cytokine released by activated mononuclear cells, induces epithelial cellmyofibroblast transdifferentiation via Jak/Stat pathway activation. J Am Soc Nephrol 2004;15:21-32.

25. Kim $\mathrm{M}$, O'Brien $\mathrm{LE}$, Kwon $\mathrm{SH}$, et al. STAT1 is required for redifferentiation during Madin-Darby canine kidney tubulogenesis. Mol Biol Cell 2010;21:3926-3933.

26. Wang J, Ouyang C, Chen X, et al. Effect of Jak2 kinase inhibition on Stat1 and Stat3 activation and apoptosis of tubular epithelial cells induced by ATP depletion/recovery. J Nephrol 2008;21:919-923.

27. Wang J, Ouyang $C$, Chen X, et al. STAT3 inhibits apoptosis of human renal tubular epithelial cells induced by ATP depletion/recovery. Nephron Exp Nephrol 2008;108:e11-e18.

28. Zhang Z, Xing J, Ma L, et al. Transglutaminase-1 regulates renal epithelial cell proliferation through activation of Stat-3. J Biol Chem 2009;284:3345-3353.
29. Horiguchi A, Oya M, Marumo $\mathrm{K}$, et al. STAT3, but not ERKs, mediates the IL-6-induced proliferation of renal cancer cells, ACHN and 769P. Kidney Int 2002;61:926-938.

30. Arakawa T, Masaki T, Hirai T, et al. Activation of signal transducer and activator of transcription 3 correlates with cell proliferation and renal injury in human glomerulonephritis. Nephrol Dial Transplant 2008;23:3418-3426.

31. Endo TA, Masuhara M, Yokouchi $M$, et al. A new protein containing an SH2 domain that inhibits JAK kinases. Nature 1997;387:921-924.

32. Starr R, Willson TA, Viney EM, et al. A family of cytokine-inducible inhibitors of signalling. Nature 1997;387:917-921.

33. Naka $T$, Narazaki $M$, Hirata $M$, et al. Structure and function of a new STAT-induced STAT inhibitor. Nature 1997;387:924-929.

34. Kile BT, Nicola NA, Alexander WS. Negative regulators of cytokine signaling. Int J Hematol 2001;73:292-298.

35. Kile BT, Alexander WS. The suppressors of cytokine signalling (SOCS). Cell Mol Life Sci 2001;58:1627-1635.

36. Larsen L, Ropke C. Suppressors of cytokine signalling: SOCS. APMIS 2002;110:833-844

37. Li M, Balamuthusamy S, Simon EE, et al. Silencing megalin and cubilin genes inhibits myeloma light chain endocytosis and ameliorates toxicity in human renal proximal tubule epithelial cells. Am J Physiol Renal Physiol 2008;295:F82-F90.

38. Ortiz-Munoz G, Lopez-Parra V, Lopez-Franco O, et al. Suppressors of cytokine signaling abrogate diabetic nephropathy. J Am Soc Nephrol 2010;21:763-772.

39. Hernandez-Vargas P, Lopez-Franco O, Sanjuan G, et al. Suppressors of cytokine signaling regulate angiotensin II-activated Janus kinasesignal transducers and activators of transcription pathway in renal cells. J Am Soc Nephrol 2005;16:1673-1683.

40. Bellezza I, Neuwirt $\mathrm{H}$, Nemes $\mathrm{C}$, et al. Suppressor of cytokine signaling3 antagonizes cAMP effects on proliferation and apoptosis and is expressed in human prostate cancer. Am J Pathol 2006;169: 2199-2208.

41. Neuwirt $H$, Puhr M, Cavarretta IT, et al. Suppressor of cytokine signalling-3 is up-regulated by androgen in prostate cancer cell lines and inhibits androgen-mediated proliferation and secretion. Endocr Relat Cancer 2007;14:1007-1019.

42. Puhr M, Santer FR, Neuwirt $H$, et al. SOCS-3 antagonises the proliferative and migratory effects of fibroblast growth factor-2 in prostate cancer by inhibition of p44/p42 MAPK signalling. Endocr Relat Cancer 2010;17:525-538.

43. Liu Q, Liu S, Shi Y, et al. Suppressors of cytokine signaling inhibit tubular epithelial cell-myofibroblast transdifferentiation. Am J Nephrol 2011;34:142-151.

44. Rudnicki $M$, Eder S, Perco $P$, et al. Gene expression profiles of human proximal tubular epithelial cells in proteinuric nephropathies. Kidney Int 2007;71:325-335.

45. Rudnicki M, Perco P, Enrich J, et al. Hypoxia response and VEGF-A expression in human proximal tubular epithelial cells in stable and progressive renal disease. Lab Invest 2009;89:337-346.

46. Puhr M, Santer FR, Neuwirt H, et al. Down-regulation of suppressor of cytokine signaling-3 causes prostate cancer cell death through activation of the extrinsic and intrinsic apoptosis pathways. Cancer Res 2009:69:7375-7384.

47. Komyod W, Bohm M, Metze D, et al. Constitutive suppressor of cytokine signaling 3 expression confers a growth advantage to a human melanoma cell line. Mol Cancer Res 2007;5:271-281.

48. Strathdee CA, McLeod MR, Hall JR. Efficient control of tetracyclineresponsive gene expression from an autoregulated bi-directional expression vector. Gene 1999;229:21-29.

49. Tonko-Geymayer S, Goupille O, Tonko M, et al. Regulation and function of the cytokine-inducible $\mathrm{SH}-2$ domain proteins, $\mathrm{CIS}$ and SOCS3, in mammary epithelial cells. Mol Endocrinol 2002;16: 1680-1695.

50. Nouwen EJ, Dauwe S, van der Biest I, et al. Stage- and segmentspecific expression of cell-adhesion molecules N-CAM, A-CAM, and L-CAM in the kidney. Kidney Int 1993:44:147-158.

51. Tsuchiya B, Sato $Y$, Kameya $T$, et al. Differential expression of $\mathrm{N}$-cadherin and E-cadherin in normal human tissues. Arch Histol Cytol 2006;69:135-145.

52. Liu KD, Brakeman PR. Renal repair and recovery. Crit Care Med 2008;36(4 Suppl):S187-S192. 
53. Weber A, Hengge UR, Bardenheuer W, et al. SOCS-3 is frequently methylated in head and neck squamous cell carcinoma and its precursor lesions and causes growth inhibition. Oncogene 2005; 24:6699-6708.

54. He B, You L, Uematsu K, et al. SOCS-3 is frequently silenced by hypermethylation and suppresses cell growth in human lung cancer. Proc Natl Acad Sci USA. 2003;100:14133-14138.

55. Barasch J, Yang J, Ware CB, et al. Mesenchymal to epithelial conversion in rat metanephros is induced by LIF. Cell 1999;99: 377-386.
56. Boccaccio $\mathrm{C}$, Ando $\mathrm{M}$, Tamagnone $\mathrm{L}$, et al. Induction of epithelial tubules by growth factor HGF depends on the STAT pathway. Nature 1998;391:285-288.

57. Li R, Yang N, Zhang $\mathrm{L}$, et al. Inhibition of Jak/STAT signaling ameliorates mice experimental nephrotic syndrome. Am J Nephrol 2007; 27:580-589.

58. Brosius 3rd FC, Banes-Berceli A. A new pair of SOCS for diabetic nephropathy. J Am Soc Nephrol 2010;21:723-724.

59. Chuang PY, He JC. JAK/STAT signaling in renal diseases. Kidney Int $2010 ; 78 \cdot 231-234$ 